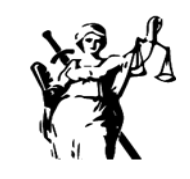

JUSTICIA

ISSN impreso 0124-7441
ISSN digital 2590-4566

\title{
La transferencia y cesión internacional de los derechos de los futbolistas profesionales y la exclusión de los terceros
}

\section{The international transfer and assignment of the rights of professional footballers and the exclusion of third parties}

\author{
(iD) \\ Walter René Cadena Afanador \\ Universidad Militar Nueva Granada, Colombia \\ walterr.cadenaa@unilibre.edu.co
}

\author{
Francisco Javier Torres Espindola \\ Universidad Libre, Colombia \\ frantosport90@gmail.com
}

Recibido: 07 de noviembre de 2019 / Aceptado: 14 de febrero de 2020 https://doi.org/10.17081/just.25.38.4586

\begin{abstract}
Resumen
El objetivo de este artículo es analizar la naturaleza jurídica y los riesgos que existen en las transferencias de los derechos de los jugadores de fútbol profesional en el ámbito internacional, así como las implicaciones de las recientes modificaciones normativas de la FIFA respecto a los clubes deportivos, los futbolistas y los terceros. Para ello, nosotros usamos un método analítico, propio de la investigación dogmática, normativa, documental, cualitativa, desde el derecho del deporte y el derecho comercial. Como resultados, se verifica que el fútbol profesional hace parte de la industria de los deportes y el entretenimiento, caracterizado por tener modelos contractuales atípicos, una estructura organizacional federativa, que se regula mediante un ordenamiento jurídico especial en el que se privilegia la normativa no estatal y que tiene un ritmo de crecimiento financiero superlativo, el cual ha configurado un campo mercantil sui generis que demanda juristas especializados en la materia. Debido a la profesionalización de los actores que intervienen, existe una regulación integrada por normas de orden público y privado, donde se destaca la normativa aprobada por la Federación Internacional de Fútbol Asociado. Se concluye que la exclusión normativa de los terceros en las transferencias y cesiones de los jugadores internacionales, así como la prohibición de figuras como el Third Party Ownership, plantean un cambio sensible en el modelo negocial y contractual en el negocio del fútbol, del que no quedan excluidos los propios jugadores, y que conlleva implicaciones respecto al financiamiento y el papel de los inversionistas en los clubes deportivos, así como de la significación de los derechos económicos de los jugadores.
\end{abstract}

Palabras clave: federación internacional de fútbol asociado, transferencia, cesión, derechos federativos, derechos económicos, terceros.

\begin{abstract}
The objective of this article is to analyze the legal nature and risks that exist in the transfer of the rights of professional soccer players in the international arena, as well as the implications of recent FIFA regulatory changes regarding sports clubs, footballers and third parties. For this, we use an analytical method, typical of dogmatic, normative, documentary, qualitative research, from the law of sport and commercial law. As a result, it is verified that professional football is part of the sports and entertainment industry, characterized by having atypical contractual models, a federative organizational structure, which is regulated by a special legal system in which non-state regulations are privileged And that has a superlative financial growth rate, it has configured a sui generis commercial field that demands specialized lawyers in the matter. Due to the professionalization of the actors involved, there is a regulation made up of public and private order regulations, where the regulations approved by the International Federation of Associated Football stand out. It is concluded that the normative exclusion of third parties in the transfers and assignments of international players, as well as the prohibition of figures such as the Third Party Ownership, pose a significant change in the business and contractual model in the football business, of which no The players themselves are excluded, and that carries implications regarding the financing and the role of investors in sports clubs, as well as the significance of the economic rights of the players.
\end{abstract}

Keywords: international federation of associated football, transfer, assignment, federation rights, economic rights, third parties

Como citar: Cadena Afanador, W. R., \& Torres Espíndola, F. J. (2020). La transferencia y cesión internacional de los derechos de los futbolistas profesionales y la exclusión de los terceros. Justicia, 25(38), 205-223. https://doi.org/10.17081/just.25.38.4586 


\section{Introducción}

Según la Federación Internacional de Fútbol Asociado (FIFA), durante el año 2019, se realizaron 18042 transferencias internacionales de jugadores masculinos de 178 nacionalidades, con un incremento del 50,3\% frente al 2012, con inversiones por 7345 millones de dólares (incremento del 76,1\% respecto al 2012) y 4162 clubes involucrados (31,3\% frente al 2012). El valor promedio de los gastos de la transferencia por jugador masculino pasó de 1,7 millones de dólares en el 2012 a 2,7 millones de dólares en el 2019. Los países con el mayor número de clubes involucrados en estas transferencias fueron Brasil (306), España (130), Inglaterra (128), Argentina (107) y Francia (97) (Federación Internacional de Fútbol Asociado [FIFA], 2020a, pp. 10-13).

En el continente americano, los mayores montos en las transferencias en el 2019 se concentraron en Brasil, México, Argentina y los Estados Unidos, representando el 79\% de las transferencias salientes (Brasil, 39\%; la Argentina, 22\%; México, 12\% y los Estados Unidos, 6\%) y el $89 \%$ de las transferencias entrantes (México, 28\%; los Estados Unidos, 27\%; Brasil, 18\% y la Argentina, 17\%). Colombia representó el 5\% de las transferencias salientes (sexto en la región) y el 1\% de las transferencias entrantes (noveno en la región) (FIFA, 2020a, pp. 28-31).

En materia de jugadoras femeninas, se presentaron incrementos en el número de transferencias (19,7\%), los clubes involucrados $(24,5 \%)$ y el monto de las transacciones $(16,3 \%)$. Los países con el mayor número de clubes involucrados en estas transferencias fueron España (29), Suecia (20), Inglaterra (19), Francia (17) y Colombia (13) (FIFA, 2020a, pp. 10-17).

En el continente americano, el mayor número de jugadoras involucradas en las transferencias en el 2019 se concentraron en seis países, representando el $84 \%$ de las transferencias salientes (Estados Unidos, 53\%; Brasil, 18\%; Venezuela, $7 \%$ y Colombia, $5 \%$ ) y el $85 \%$ de las transferencias entrantes (Estados Unidos, $43 \%$; Colombia, 24\%, México, 11\%; y la Argentina, 7\%). (FIFA, 2020a, pp. 28-31).

La industria del deporte y el entretenimiento vinculada con el fútbol profesional ha tenido un crecimiento notorio en las últimas cuatro décadas, habida cuenta del impacto cultural y social que genera el espectáculo, adicional a los grandes intereses publicitarios y comerciales que le rodean que ha erigido a la FIFA en una organización no gubernamental de connotaciones corporativas globales, como pocas. Los clubes deportivos se han convertido en destacadas empresas, cuya razón social van más allá del cumplimiento de su objeto en materia deportiva, puesto que ejercen actividades de comercio a través de diferentes mecanismos de financiación, como la venta de tiquetes para los partidos de fútbol, cesiones de derechos de televisión e imagen tanto de los clubes como de los deportistas, venta de artículos deportivos, posicionamiento de sus marcas en el mercado, entre otros aspectos.

Para el 2019, los 36 clubes de fútbol colombianos adscritos a los torneos de primera y segunda división son sociedades anónimas en su mayoría, aunque también hay corporaciones y asociaciones, los cuales generaron ingresos operaciones por $\$ 648$ mil millones (incremento del $13,6 \%$ frente al 2018). El $78,4 \%$ de estos ingresos correspondieron a los derechos deportivos (26,1\%), derechos de televisión Dimayor (19,3\%), venta de boletería (16,7\%) y patrocinio y publicidad (16,3\%) (Superintendencia de Sociedades, 2020, pp. 7-12). De los gastos operacionales, el $18,6 \%$ se refirieron a amortización de derechos deportivos y venta de derechos deportivos (Superintendencia de Sociedades, 2020, p. 15), generándose un ejercicio financiero deficitario por $\$ 21,7$ mil millones en los equipos de la primera división (Superintendencia de Sociedades, 2020, pp. 30-31). 
En la industria del fútbol, además de los ingresos corrientes que perciben los clubes, se encuentran las transferencias de futbolistas profesionales de un club a otro, mecanismo que se usa para suplir las plantillas de jugadores de los clubes, posicionar las grandes estrellas de este deporte como marcas publicitarias e incrementar sus éxitos gracias a las habilidades tácticas y técnicas de los jugadores. Estas transferencias se convierten en un importante ingreso para los clubes en Sudamérica, por cuanto no poseen la infraestructura técnica, deportiva y financiera de los clubes de Europa, presentándose un desequilibrio en la competencia deportiva. Aquellos clubes usualmente se ven forzados a vender sus mejores jugadores a los clubes con mayor poder adquisitivo en el mercado.

Por lo anterior, este trabajo realiza un análisis de los aspectos relacionados con las transferencias de futbolistas, a partir de su naturaleza jurídica, riesgos, implicaciones financieras y prohibiciones, tomando como base la normativa expedida por la FIFA, en especial, el Reglamento sobre el Estatuto y Transferencia de Jugadores. Con una metodología descriptiva, normativa, dogmática, documental y cualitativa, se procura indagar acerca del problema jurídico de cuáles son las implicaciones jurídicas que tienen las enmiendas aprobadas por la FIFA en 2015 y 2019 al Reglamento sobre el Estatuto y Transferencia de Jugadores.

\section{Transferencias internacionales de jugadores y derechos federativos}

Los Estatutos de la FIFA (2016), así como sus reglamentos, son los ejes normativos que regulan el fútbol organizado a nivel mundial. De ellos, se derivan las regulaciones en materia de gobernanza, reglamentación de competiciones y del juego, lucha contra el dopaje, ética deportiva y transferencias. Sobre este último componente, se destaca el Reglamento sobre el Estatuto y Transferencia de Jugadores (RETJ), como normativa específica para transferencias internacionales. Las transferencias de derechos de jugadores, entre clubes de una misma asociación están sujetas a una reglamentación particular emanada desde cada asociación, la cual será aprobada por la FIFA (FIFA, 2019a, art. 1, num. 2).

Los actores jurídicos esenciales en la transferencia internacional de futbolistas, requiere que se diferencie entre jugadores aficionados y jugadores profesionales:

1. Los jugadores que forman parte del fútbol organizado son aficionados o profesionales.

2. Un jugador profesional es aquel que tiene un contrato escrito con un club y percibe un monto superior a los gastos que realmente efectúa por su actividad futbolística. Cualquier otro jugador se considera aficionado. (FIFA, 2019a, art. 2)

El Reglamento establece, como regla general, que un futbolista profesional siempre debe suscribir un contrato escrito con la entidad deportiva. Si a un jugador se le paga por su actividad deportiva, incluyendo la cancelación de primas y demás beneficios económicos, si no ha suscrito un contrato por escrito, no se le considerará, en principio, un jugador profesional. Será considerado, entonces, como un jugador aficionado que ejerce la actividad deportiva por mero pasatiempo, donde los emolumentos recibidos por parte del club en donde juega son un incentivo económico por el tiempo invertido, mas no por una actividad profesional. 
Los beneficios económicos obtenidos por una transferencia internacional o por la cesión de los derechos federativos solo se atribuyen a un jugador profesional de fútbol, derivados de la transferencia de derechos federativos en el fútbol profesional.

\section{Los derechos federativos}

El objeto en el negocio jurídico de las transferencias de futbolistas son los derechos federativos, los cuales son de propiedad exclusiva de los clubes, al ser los titulares de la facultad exclusiva de registrar al jugador en la asociación nacional en la cual se encuentra afiliado el club. Así, "estos derechos son derivados del fichaje del jugador y se sitúan en la potestad que tiene el club de registrarlo en una asociación nacional, con el fin que represente a dicho órgano en una competición oficial" (Palazzo, 2014, p. 1). Cuando se presenta la transferencia de un futbolista profesional, se transfiere la posibilidad de que un club inscriba al jugador adquirido en su asociación y pueda competir deportivamente en un torneo regional, nacional y/o internacional.

La legislación deportiva colombiana prevé que los derechos federativos son llamados derechos deportivos, los cuales son poseídos únicamente por los clubes deportivos profesionales y aficionados, excluyendo a las personas naturales o jurídicas distintas del club poseedor (Ley 181 de 1995, art. 32, Col).

Los derechos deportivos son definidos por vía jurisprudencial (CC, Sentencia C-320 de 1997, Col.; CC Sentencia T-498/94, Col.; CC, Sentencia T-302/98, Col.; CC, Sentencia T-371/98, Col.) como la titularidad exclusiva de un club de transferir esta clase de derechos, recibiendo las debidas indemnizaciones por la formación y promoción del jugador:

Este tipo concreto y especial de cesión de derechos deportivos es difícil enmarcarlo dentro de la normatividad civil o comercial colombiana debido a la naturaleza misma de los derechos que se ceden o están implícitos en el negocio, como lo es el control de la actividad de los jugadores, en nuestro caso de los futbolistas. Es así como el objeto sobre el cual recae la cesión hace referencia a la actividad humana, enmarcándose como una relación jurídica atípica (CC, Sentencia T-302/98, Col.).

Los derechos deportivos son la consecuencia de la titularidad de determinado club para registrar, inscribir o autorizar a un futbolista en un torneo (Ley 181 de 1995, art. 34, Col), "siempre y cuando se entienda que se debe contar con el consentimiento del deportista y no se puede desmejorar su situación laboral" (CC, Sentencia C-320 de 1997, Col.). Ningún deportista puede ser dueño de sus derechos federativos, siendo el club propietario de la licencia federativa del jugador, la cual es otorgada por la asociación nacional respectiva, siendo el único dueño de los derechos deportivos.

Una vez las partes acuerden una transferencia internacional, el club dueño de la licencia federativa, deberá cederla sin condiciones a la asociación nacional en la cual el club cesionario se encuentre registrado, a través del certificado de transferencia internacional (CTI).

\section{La transferencia de derechos de futbolistas profesionales}

El RETJ no incorpora un concepto claro sobre qué es una transferencia de derechos en el fútbol profesional por debe acudirse a la interpretación normativa. Se entiende que es un acto jurídico, es decir, la manifestación de voluntades, capaz de producir efectos en derecho, como es crear, modificar o extinguir 
una relación jurídica. Es de carácter plurilateral, toda vez que en el acto intervienen, al menos, tres partes: el club cedente, el club cesionario y, el jugador profesional, quien debe manifestar de forma inequívoca su voluntad de ser cedidos sus derechos federativos.

(...) una transferencia es un acto jurídico complejo, toda vez que, al intervenir las voluntades de los sujetos anteriormente descritos, se observa, un contrato de trabajo vigente, una oferta del club de destino que pretende contratar los servicios del jugador y, la más importante la voluntad tacita o escrita del jugador de aceptar las condiciones contractuales en dicho negocio jurídico, sin ninguna de las tres vertientes jurídicas coinciden de manera simultánea, el acto será ineficaz. (Auletta, 2014, p. 130)

La transferencia de derechos genera obligaciones, donde la principal es la cesión definitiva o temporal de los derechos federativos de un jugador de fútbol profesional, mientras que las obligaciones accesorias, son la cesión de derechos de imagen, publicitarios y económicos, donde este tipo de contrato:

(...) no solo conlleva la realización de un acto jurídico de naturaleza plurilateral sino también de varios actos de naturaleza jurídica, en primer orden, la rescisión de un contrato laboral, por otro lado, la celebración de un "contrato de transferencia" entre los clubes en los cuales se va a transferir los derechos federativos, y en última instancia la celebración de un nuevo contrato laboral entre el club cesionario y el jugador (Auletta, 2014, p. 130)

Se configuran, entonces, tres actos jurídicos simultáneos, como son la terminación de un contrato laboral, la realización de un contrato de transferencia de derechos federativos y el surgimiento de un nuevo contrato laboral. Esta simultaneidad, usualmente es concurrente debido a la naturaleza jurídica plurilateral del negocio comercial.

\section{Tipos de transferencias de jugadores}

Las transferencias de los derechos de los futbolistas profesionales, según la regulación de la FIFA, pueden ser definitivas o temporales. Se entenderá por transferencia definitiva aquella que el club cedente traspasa al club cesionario, de manera total y permanente los derechos federativos del jugador, donde este club pagará una cantidad de dinero por la rescisión del contrato que vinculaba al jugador con el club de origen. Se requiere no solo el acuerdo de voluntades de ambas partes, sino que debe concurrir el desembolso del pago de la cláusula de recisión, en el evento de que se haya pactado, el cual corresponde al coste de los derechos federativos del jugador.

Por su parte, la transferencia temporal se configura como el préstamo, por un plazo determinado o determinable, de los derechos federativos del jugador a un club cesionario, el cual asumirá los gastos contractuales y prestacionales del jugador:

Un jugador profesional puede cederse a otro equipo en calidad de préstamo sobre la base de un acuerdo por escrito entre el jugador y los clubes en cuestión. Cualquier préstamo está sujeto a las mismas disposiciones que se aplican a la transferencia de jugadores, incluidas las estipulaciones sobre la indemnización por formación y el mecanismo de solidaridad. (FIFA, 2019a, art. 10.1)

La transferencia temporal de los derechos no rescinde el contrato laboral existente entre el futbolista y el club de cedente, ya que solo opera la suspensión de los efectos contractuales por el término pactado de la cesión. Este tipo de transferencia podrá pactarse a título gratuito u oneroso, conforme al acuerdo entre las tres partes. También se puede incluir una cláusula de opción de compra, por la cual se le otorga al club 
cesionario un orden preferencial para adquirir los derechos federativos del jugador, una vez culminen los efectos de la transferencia temporal.

\section{El contrato de transferencia internacional}

El objeto contractual de este acto jurídico plurilateral es la cesión de derechos federativos como fuente primaria de la obligación. Se trata de un negocio jurídico que involucra la transferencia de un bien intangible del club cedente, mas no del jugador como si fuese un bien, por lo que no es comparable este acto comercial con otros actos ilegales como la trata de personas o la esclavitud. Tampoco se hace la transferencia de un contrato laboral, toda vez, que el mismo se debe rescindir o terminar, antes de ejecutar la transferencia, con el fin de darle vida jurídica a una nueva relación contractual entre el jugador cedido y el club de destino o cesionario.

De manera práctica, lo que se transfiere en este contrato son los derechos federativos de un jugador, es decir, la facultad que tiene un club de registrar a un jugador ante una asociación nacional, para que este pueda representarlo en las competiciones nacionales e internacionales en las que el club lo haya inscrito oportuna y efectivamente. El contrato de transferencia es consensual, por cuanto sus efectos dependen de lo acordado por las partes, siempre por escrito.

Luego de la firma del contrato por las tres partes en cuestión, éste no podrá ser incumplido, so pena de ejercer acciones de tipo ejecutivo para el cobro de sus cláusulas penales, las cuales que se podrán ejercer únicamente ante la Comisión del Estatuto del Jugador en primera instancia y ante la Cámara de Resolución de Disputas de FIFA, en segunda instancia, o ser elevado ante el Tribunal de Arbitramento Deportivo (TAS), al ser un conflicto de orden internacional y federativo.

Cuando se perfecciona un contrato de transferencia internacional de derechos federativos, se debe expedir automáticamente y sin condicionamientos el Certificado de Transferencia Internacional por parte de la asociación nacional a la que está afiliado el club cedente:

Los jugadores inscritos en una asociación únicamente podrán inscribirse en una nueva asociación únicamente cuando esta última haya recibido el certificado de transferencia internacional (en adelante, «el CTI») de la asociación anterior. El CTI se expedirá gratuitamente, sin condiciones ni plazos. Cualquier disposición en contra se considerará nula y sin efecto. La asociación que expide el CTI remitirá una copia a la FIFA. Los procedimientos administrativos para la expedición del CTI se encuentran definidos en el anexo 3 , art. 8, y en el anexo 3a del presente reglamento. (FIFA, 2019a, art. 9.1)

La asociación nacional en la que el club cesionario se encuentra registrado debe solicitar a la asociación del club cedente, la expedición del CTI, con el visto bueno de dicho club, con el fin de registrar los derechos deportivos derivados de la licencia federativa, para que de esta manera el jugador transferido pueda competir. Sin el visto bueno del club de origen, no se podrá formalizar el negocio jurídico.

\section{Características de los derechos federativos}

Los derechos federativos o deportivos de un futbolista están a disposición de los clubes afiliados a una asociación nacional, que para que pueda hacer uso de ellos, requiere tanto de la existencia de un contrato laboral con el jugador, como de la transferencia de los derechos federativos y su inscripción en un registro avalado por la asociación correspondiente. Estos derechos federativos 
Se originan a partir de una licencia federativa sin supeditarse al contrato de trabajo, es decir, tendrán efectos jurídicos una vez que club de destino registre tales derechos ante su asociación nacional, no basta entonces con la suscripción de un contrato laboral, toda vez que si no se registran tales derechos el jugador queda inhabilitado para representar al club de destino en una competición organizada.

Los derechos federativos son de propiedad exclusiva de clubes legalmente reconocidos y registrados ante una asociación nacional, por lo que ninguna persona natural o jurídica puede poseerlos, ni siquiera el mismo jugador (Auletta, 2014, p. 20)

La titularidad de los derechos deportivos recae en los clubes deportivos, cuyos rendimientos económicos no pueden ser cedidos a otras personas físicas o jurídicas. El procedimiento para inscribir los jugadores debe cumplir los requisitos previstos por la Federación correspondiente, esto es, la manifestación clara de aceptación del deportista, la transferencia de la ficha o licencia deportiva y la existencia de un contrato de trabajo. En el caso colombiano, el contrato debe ser registrado en la Federación Colombiana de Fútbol y en Coldeportes (Ley 181 de 1995, art. 32, Col). Así, los derechos federativos son indivisibles por cuanto

(...) esta clase de derechos no pueden ser compartidos por dos clubes o personas físicas o jurídicas, por lo que en el ordenamiento jurídico deportivo internacional, ningún jugador puede representar a dos instituciones deportivas a la vez, en un mismo torneo o temporada, por ende, los derechos federativos le pertenecen como se expresó anteriormente a un solo club.

Por último, los derechos federativos deben ser transferidos de manera íntegra más no parcial, por lo que tiene conexidad con las características de que los mismos son indivisibles (Auletta, 2014, p. 22)

La cesión en una operación económica, como la transferencia de futbolistas, abarca un contenido económico, siendo también una característica del derecho federativo, el cual representa un ingreso o un gasto de los clubes convierte, el cual se complementa con las comisiones acordadas con el jugador y su agente intermediario, lo cual es denominado por la doctrina deportiva como los derechos económicos.

\section{Cesión internacional de jugadores y derechos económicos}

Es la retribución pagada en dinero por parte del club cesionario al club cedente por la transferencia o cesión de los derechos federativos de un jugador. Los derechos económicos son el valor que se atribuye en el mercado por la valoración de un futbolista, sin confundirlo como un objeto, sino como el valor activo de un club representado en unos derechos federativos. Se trata de un producto del mercado de la industria del fútbol, surgida en Sudamérica, particularmente, en la Argentina y desarrollada masivamente en Brasil (Gerbaundo, 2019)

Los derechos económicos "se refieren a la vertiente patrimonial de los derechos federativos y para su existencia es preciso que, junto a la inscripción federativa, exista un contrato laboral que rija la relación entre el club y el deportista" (Cazorla, 2016, p.1), los cuales surgen del valor de la transferencia de un club a otro, al ser el valor económico de los derechos federativos (Bruera, 2018).

Los derechos de contenido patrimonial le pertenecen únicamente a los clubes y a los jugadores, siempre y cuando estos tengan un vínculo laboral vigente con el club, por ende. En Colombia es prohibido ceder los derechos económicos a personas distintas a los clubes (Ley 181 de 1995, art. 32, Col). 
Los derechos económicos pueden ser tasados por los estudios de mercado en los que se valoran las estadísticas y el rendimiento deportivo del jugador, por mutuo acuerdo entre las partes conforme a dichas variables, o por la obligación de ejecutar una cláusula de recisión que ha sido previamente pactada en el contrato laboral del jugador. En este caso, la cláusula contiene un valor económico que debe ser pagado por el club que se encuentre interesado en los servicios del jugador y que, al hacerla efectiva, el futbolista podrá ser transferido. Esa cláusula da lugar a la indemnización de daños y perjuicios ocasionados por la ruptura de un contrato antes del vencimiento pactado.

Los jugadores de fútbol profesional pueden ser copropietarios de sus derechos económicos, con base en un porcentaje pactado con el club o los clubes dueños de los mismos. Así, podrán tener un beneficio patrimonial al ser transferidos a determinado club, mediante el pago de una prima económica.

A diferencia de los derechos federativos, los derechos económicos sí son divisibles, pudiéndose fraccionar conforme al acuerdo pactado entre las partes, configurándose la cotitularidad. Este tipo de derechos es un mecanismo de enriquecimiento legítimo para los clubes, ya que, a través de la venta de derechos federativos, estos pueden funcionar como clubes deportivos o sociedades anónimas. Se trata de unos derechos derivativos, ya que se desprenden de los derechos deportivos o federativos.

Los derechos económicos pueden ser negociados entre clubes mediante la cesión de derechos económicos, en la que se crea una expectativa condicionada para obtenerlos, una vez se perfeccione la transferencia de derechos federativos en un lapso posterior a la misma.

A diferencia de los derechos deportivos, en los derechos económicos se podía pactar la cotitularidad a terceras personas físicas o jurídicas, ajenas a la negociación de la transferencia del futbolista. Se trataba de un contrato de cesión de derechos económicos que podía involucrar a personas naturales o jurídicas diferentes a los clubes. Sin embargo, la FIFA prohibió esta modalidad en la cesión de los derechos económicos a partir del 2015, declarando la interdicción e invalidez de los contratos que no fuesen modificados. La RETJ en el artículo 18 ter

\begin{abstract}
(...) contiene la prohibición de que los clubes y los jugadores celebren acuerdos con terceros, por los que el tercero tuviera derecho a participar en una compensación pagadera en relación con la futura transferencia de un jugador, o que se le asigne cualquier derecho en relación con una futura compensación por transferencia. (FIFA, 2014)
\end{abstract}

\title{
El contrato internacional de cesión de derechos económicos
}

En Colombia el mecanismo idóneo para realizar una transferencia definitiva o temporal de un jugador, así como autorizar su inscripción en un nuevo club, es el convenio, nombre que la citada ley le ha otorgado al contrato de compraventa que permite la cesión de los derechos económicos y deportivos (Ley 181 de 1995, art. 35, Col).

A partir de la década de 1990 se incrementó el número y el valor de las transferencias de los jugadores sudamericanos a los clubes europeos, significando un debilitamiento en el perfil de los futbolistas en los clubes de la región, incentivando la búsqueda de estrategias para no perder la inversión en la formación de jugadores talentosos, así como fortalecer los procesos de formación de futbolistas que pudiesen servir de recambio en la plantilla y que después pudiesen ser transferidos. 
De esta forma surgió la cesión de derechos económicos a terceros en calidad de inversionistas, quienes podían ser personas naturales o jurídicas. Se trataba de un negocio jurídico mediante el cual, el club propietario de los derechos federativos de un jugador cede los beneficios económicos al club de destino del jugador y/o a un tercero inversor, con el objeto de que la cantidad pactada sea efectiva ante una futura transferencia de sus derechos federativos:

El contrato de cesión de derechos económicos derivados de la transferencia de derechos federativos sobre un jugador de fútbol es un contrato en el cual, el club cede a un inversor los beneficios económicos futuros, condicionales y aleatorios derivados de la venta o préstamo de los derechos federativos de un deportista (Di Ángelo, 2015).

El tercero inversor podía percibir un monto determinable como retribución a su contribución en la formación del jugador, o a su manutención dentro del club, o por haber prestado un servicio técnico o asistencial a modo de inversión:

(...) en su modalidad básica y más frecuente, el club celebra un contrato con uno o varios "terceros" (inversores, empresarios, grupos económicos, etc.) por medio del cual, a cambio de un precio, cede a ese tercero inversor un porcentaje del dinero (beneficio económico) que puede llegar a recibir en el futuro, como resultado de la transferencia de los derechos federativos de un determinado jugador en su plantel a otro club (Auletta, 2014, p. 133).

\section{La validez del contrato de cesión de derechos económicos}

Un elemento esencial en este tipo de contrato es la cotitularidad, ya que los derechos económicos pueden ser compartidos y divididos con otros clubes, por lo que podrán ser transferidos, siempre y cuando el jugador tenga una relación contractual con alguno de los clubes contratantes e intervenga el consentimiento del mismo.

La propiedad de los derechos económicos se podrá compartir, situación que es válida según la normativa de la FIFA y reconocido por el Tribunal de Arbitramento Deportivo, siempre que cumpla los requisitos indicados y que el club que ostente los derechos federativos del jugador dé el visto bueno a la transferencia del jugador (Crespo \& Frega, 2015). No existirá enriquecimiento sin justa causa que invalide este contrato, por lo que la distribución de los derechos económicos dependerá de lo pactado por las partes:

(...) un jugador únicamente puede ser registrado para jugar en único club, nunca con dos o más al mismo tiempo, por lo que la transferencia parcial de sus derechos federativos contraviene la esencia de la normativa FIFA y es además imposible. Sin embargo, nada obsta que dos clubes decidan realizar negocios sobre los "derechos económicos" de un jugador, en tanto que el jugador preste su consentimiento y mantenga una relación laboral con alguno de los clubes implicados pues, por ejemplo, un club que no puede ceder en ningún caso - como ya se ha dicho - una parte de los derechos federativos de un jugador que, como un todo, solo pueden estar registrados a favor de un único club, sí que puede ceder a otro una parte de los derechos económicos - que no se registran y que forman parte de su patrimonio - derivados por ejemplo de sus relaciones contractuales con algún club de fútbol.

Como consecuencia de ello, si bien los "derechos federativos" de un jugador no pueden ser compartidos entre dos clubes, los "derechos económicos" de un jugador si pueden ser objeto de cotitularidad y, por tanto, parcialmente transferidos. (Crespo \& Frega, 2015, p.p 246 - 247) 
La cesión de derechos económicos debe hacerse a título oneroso por cuanto la inversión que se realiza es de tipo económico, técnico o asistencial, donde el objeto contractual debe ser lícito, sin afectar los derechos fundamentales ni la voluntad del jugador. Conforme a la normativa comercial colombiana, este contrato se asemeja a la cesión de contratos (Decreto 410 de 1971, art. 887, Col), teniendo en cuenta la naturaleza mercantil, con una ejecución instantánea una vez se surta la compraventa de los derechos deportivos del jugador. Se trata de un contrato celebrado intuitu personae por clubes deportivos y, que deberá concurrir con la aceptación del futbolista.

\section{Características del contrato de cesión de derechos económicos}

Las principales características del contrato de cesión de derechos económicos de un futbolista son:

- Plurilateralidad. En este contrato deben converger, al menos, tres partes para su celebración: el club propietario y que cede los derechos federativos del jugador, el club cesionario que funge como tercero inversor, con el cual se compartirán los beneficios económicos en el evento de una futura transferencia del jugador y, el jugador, quien no solo manifiesta su voluntad, sino que percibirá parte de los beneficios de la transferencia de los derechos económicos.

- Consensualidad. El contrato se perfecciona por el consentimiento manifiesto y escrito de las partes.

- Aleatoriedad. El perfeccionamiento de este contrato depende de un hecho futuro e incierto, que es la transferencia de los derechos federativos del jugador.

- Accesoriedad. Por un lado, el contrato de cesión de cesión de derechos económicos es posterior a la transferencia de los derechos federativos del futbolista y, por otro lado, su celebración se garantiza con el pago de una acreencia pactada, la cual será obtenida por la futura venta de los derechos federativos.

- Onerosidad. El club dueño de los derechos federativos del jugador deberá pagar una suma dineraria por los porcentajes pactados previamente en el contrato de cesión, al club cesionario y/o al club que funge como tercero inversor, como resultado de la venta de los derechos deportivos.

- Frecuentemente mercantil. Es un contrato que usualmente es de derecho comercial y mercantil, ya que muchos de los clubes involucrados en este tipo de tratativas cuentan con registro mercantil. En Colombia, a diciembre de 2019, la mayoría de los clubes deportivos han sido constituidos como sociedades anónimas, sujetos a registro y con capacidad para ejercer actos de comercio. En la primera división, 18 de los 20 clubes son sociedades anónimas (el Deportivo Cali y el Deportivo Pasto son asociaciones), mientras que, en la segunda división, 14 de los 16 clubes son sociedades anónimas (el Cortuluá y el Deportivo Pereira son corporaciones, esta última, en proceso de liquidación) (Superintendencia de Sociedades, 2020, pp. 7-8).

- Internacionalidad. Tratándose de la transferencia internacional de un jugador de fútbol profesional, los clubes involucrados deben pertenecer a asociaciones nacionales diferentes. Ello no excluye la posibilidad que se formulen contratos de cesión de derechos económicos en transferencias domésticas o nacionales de futbolistas.

- Es típico. Por aplicación analógica de la normativa colombiana, esta clase de contrato se asimila a la cesión de créditos, regulada por el Código de Comercio (Decreto 410 de 1971, art. 887, Col.). La normatividad federativa de la FIFA no define esta clase de contrato, pero sí los regula, imponiendo recientemente una serie prohibiciones y limitaciones a los terceros intervinientes que no son clubes deportivos. 


\section{Obligaciones de las partes en los contratos de cesión de derechos económicos}

Por tratarse de un contrato de naturaleza mercantil, se rige por el principio lex contractus, pacta sunt servanda (Ley 84 de 1873, art. 1602, Col.), atribuyendo las siguientes obligaciones a las partes en una transferencia internacional.

\section{Obligaciones del club deportivo cedente}

- Comunicar las ofertas recibidas para la compra de los derechos deportivos del jugador a este, así como a los clubes que ofician como terceros inversores.

- Tener en cuenta y respetar el consentimiento del futbolista frente a la oferta de transferencia de sus derechos federativos.

- Ceder al club cesionario los beneficios económicos pactados en el momento en que se produzca la transferencia del jugador a un club afiliado a otra federación.

- Ceder los porcentajes estipulados en un contrato de cesión de derechos económicos, a un club inversionista, como resultado de la transferencia de los derechos federativos del jugador.

- Pagar al jugador los derechos económicos pactados.

- Cumplir con las obligaciones laborales hasta el momento que se realice efectiva la transferencia de los derechos federativos del jugador.

- Obligaciones del club deportivo cesionario

- Pagar el precio previsto en el contrato de cesión de los derechos económicos, dentro de los plazos y montos acordados.

- Coadyuvar con el club cedente en los gastos de representación del jugador, relativos a su proceso de promoción y formación.

- Gestionar los mecanismos idóneos para ofertar los derechos deportivos del jugador en el mercado futbolístico, a un precio razonable, que les brinde ganancias a las partes intervinientes.

\section{Obligaciones del futbolista}

- Otorgar su consentimiento libre y expreso para ser transferido a otro club, conforme a la oferta presentada por el club pretendiente y comunicada debida y oportunamente por el club titular de los derechos federativos. Con ello se debe buscar la garantía de la estabilidad laboral y la mejora de las condiciones económicas y/o deportivas del jugador.

En el contrato de cesión de derechos económicos, tanto el club cesionario como los clubes inversionistas no podrán exigir el cumplimiento del mismo en materia de pagos o indemnizaciones a su favor, así haya finalizado el término pactado contractualmente, cuando no se logró realizar la venta de los derechos federativos del jugador. Sin embargo, puede caber la excepción de que se demuestre la indebida diligencia

en las obligaciones o la mala fe del club cedente o del club propietario de los derechos federativos, en no haber realizado la transferencia del futbolista. 


\section{La propiedad de los terceros respecto a los derechos económicos}

Un tercero es toda persona natural y/o jurídica, distinta al jugador sujeto de la transferencia, a los dos clubes sobre los cuales se realiza la transferencia de los derechos del jugador, o a los clubes a los que perteneció el jugador objeto de la operación económica (FIFA, 2019a, Definiciones num. 14).

A partir de la década de 1990 hizo carrera, en especial en Iberoamérica, la figura de los Third Party Ownership (TPO), los cuales eran inversionistas privados que adquirían parte de los derechos económicos de los futbolistas, lo que les generaba réditos en la futura transferencia de los derechos deportivos de un jugador. Así, un TPO podía adquirir por \$200 000 dólares el 50\% de los derechos deportivos del jugador en Colombia, en copropiedad con el club titular de los derechos federativos. Cuando dichos derechos fuesen transferidos a otro club de la misma federación o de otra federación por un valor de \$4000 000 dólares, el TPO recibiría \$2 000000 por su parte en los derechos deportivos (Batista, 2017).

- La FIFA, mediante la Circular núm. 1464 (FIFA, 2014a) prohibió a partir de 2015 la cesión de los derechos económicos en favor de terceras personas que son ajenas al mercado del fútbol. Esta decisión fue corroborada por el Tribunal Arbitral del Deporte (TAS, 2017, RFC Seraing vs. FIFA), el cual consideró que eran justificados los argumentos presentados por la FIFA, con base en dos premisas:

- Evitar la influencia de terceros en las decisiones deportivas y económicas de los clubes deportivos, y de esta manera, evitar un conflicto de intereses entre las partes durante la transferencia de los derechos federativos de un futbolista profesional (FIFA, 2019a, art. 18 bis).

\section{El dinero que es del fútbol se debe quedar en el entorno del fútbol.}

Conforme a la reciente normativa de la FIFA, se prohíbe a los TPO, que fungían como terceros inversores en este tipo de contratos, intervenir en las políticas laborales y deportivas de los clubes cedentes o cesionarios, procurando que se respetara su autonomía e independencia financiera, corporativa y deportiva. En la práctica existía un alto riesgo de indebida presión (Cazorla, 2014) e injerencia de estos terceros inversores y sus agentes en dichos clubes.

En gran medida, los riesgos que se evidencian en la práctica de los TPO, se deben a que la FIFA no puede ejercer control alguno, ya que los TPO no están afiliados a la organización ni a las federaciones nacionales. Algunos de los riesgos inherentes a la injerencia de los TPO en la transferencia de derechos de jugadores, son los siguientes:

- Opacidad en los inversores involucrados que pueden escapar a la supervisión, tanto de los organismos reguladores del fútbol como de las autoridades estatales, al realizar negocios de forma descontrolada. Muchas veces es imposible conocer los verdaderos accionistas de los fondos de inversión y la naturaleza de sus inversiones, los cuales operan como empresas off-shore o de fachada en países que son catalogados como paraísos oficiales, o tienen una regulación fiscal que se les asemeja. Otras veces, los TPO cuentan con co-contratistas, quienes ceden y transfieren sus derechos contractuales a terceros anónimos.

- Vulneración de la libertad profesional y de los derechos económicos de los jugadores al interponerse un interés especulativo en la transferencia

- Conflictos de intereses, fraude o manipulación de partidos, ya que el mismo inversor puede ser un TPO en varios clubes pertenecientes a la misma competición, afectando la integridad y deportividad de los torneos. 
- Riesgos éticos ya que el objetivo principal del TPO es financiero y especulativo, dejando de lado los objetivos deportivos, sociales y, hasta culturales, que acompañan al fútbol como ese fenómeno masivo en que se ha convertido (TAS, 2017, RFC Seraing vs. FIFA, nums. 108 y 117, pp. 20 y 22).

De esta manera, todo contrato de cesión de derechos económicos celebrado con un tercero será ineficaz, quedando los clubes y los jugadores intervinientes in curso de eventuales sanciones. La Comisión Disciplinaria de la FIFA vela por el estricto cumplimiento de las obligaciones y prohibiciones contenidas en el RETJ, estando facultada para imponer las sanciones deportivas y administrativas que estime pertinentes (FIFA, 2019a, arts. 18 bis num. 1 y 18 ter num. 6).

\section{¿Es legalmente válida la prohibición de la FIFA a los TPO?}

En 2017 el TAS decidió una controversia arbitral que implicó a un extinto club belga contra la FIFA, a propósito de la cesión de derechos de tres jugadores en la que estaba involucrada una empresa maltea como TPO, motivo por el cual la Comisión Disciplinaria de la FIFA abrió investigación disciplinaria contra el club belga por infracciones cometidas a los artículos 18 bis y 18 ter del RETJ (TAS, 2017, RFC Seraing vs. FIFA).

Este laudo arbitral es muy importante ya que estudia la legalidad de las normas expedidas por la FIFA frente al Tratado de Formación de la Unión Europea (TFUE) y la Carta de Derechos Fundamentales de la Unión Europea (CDFUE), en especial, frente a la libre circulación de trabajadores (UE, 2007, art. 45), la libertad de prestación de servicios (UE, 2007, art. 56), la libertad de circulación de capital (UE, 2007, art. 63), la libertad profesional y laboral, así como la libertad de empresa (UE, 2000, arts. 15 y 16). En un extenso análisis, el TAS concluyó que las modificaciones aprobadas por la FIFA al RETJ no vulnera:

- La normativa europea relativa a la libre circulación de capitales

- La libre circulación tanto de los trabajadores como de la prestación de servicios

- El derecho de la competencia de la Unión Europea

Frente al primer aspecto, el tribunal consideró que el artículo 18 bis del RETJ (FIFA, 2019a) no es discriminatorio cuando se aplica el apartado "los club(es) contrario(s) y viceversa o a terceros" (FIFA, 2019a), por cuanto no se aplica a todos sin distinción (TAS, 2017, RFC Seraing vs. FIFA, num. 98, p. 18). Si bien el TAS reconoce que el RETJ plantea obstáculos para algunas libertades protegidas por el TFUE, no por ello "constituyen obstáculos no necesariamente prohibidos" (TAS, 2017, RFC Seraing vs. FIFA, num. 100, p. 18), ya que el Tribunal de Justicia de la Unión Europea ha conceptuado en reconocidos fallos como los casos «Bosman» (TJCE, 1995, Union Royale Belge des Sociétés de Football Association ASBL vs. Jean-Marc Bosman et. al.) o «Kraus» (TJCE 1993, Dieter Kraus vs. Land Baden-Wuerttemberg) que estas limitaciones son admisibles siempre que se persiga un objetivo legítimo y se justifique por razones imperiosas de interés general, circunscribiéndose a estos objetivos.

En este caso, el TAS evidenció que los objetivos perseguidos por la FIFA al aprobar estas enmiendas del RETJ son legítimos para evitar el trato discriminatorio en la transferencia de los futbolistas (TAS, 2017, RFC Seraing vs. FIFA, num. 120, p. 23), por cuanto buscan

(...) la preservación de la estabilidad de los contratos de los jugadores, la garantía de la independencia y autonomía de clubes y jugadores en términos de contratación y transferencias, la salvaguarda de la integridad en el fútbol y el carácter justo y equitativo de las competiciones, previniendo conflictos de interés y manteniendo la transparencia en las transacciones relacionadas con transferencias de jugadores. (TAS, 2017, RFC Seraing vs. FIFA, num. 100, p. 19) 
Con relación a la libre circulación de trabajadores y la libertad de prestación de servicios, el TAS planteó argumentos similares a los ya anotados frente a la libre circulación de capitales, además que la parte recurrente no logró demostrar una caída en el número de transferencias de jugadores intraeuropeos, así como una caída en el monto de financiación e inversión en la industria del fútbol, generando un hipotético efecto deflacionario en el valor de las transferencias de los derechos económicos.

Frente a la legalidad de los mencionados artículos del RETJ frente al derecho de la competencia de la Unión Europea, el TAS evaluó si se con esta normativa se estimulaba la formación de un cartel prohibido y si se vulneraba el TFUE (UE, 2007, art. 101), al configurarse un posible monopolio en las transferencias y cesiones en favor de los clubes deportivos, al excluir a otros terceros. Para ello, el panel arbitral analiza los requisitos que deben concurrir para que se configure una actuación anticompetitiva:

- Que exista un acuerdo entre empresas

- Que las actuaciones o medidas afectan el comercio de los Estados miembros de la Unión Europea

- Que la medida busque restringir, prevenir o falsear la competencia en el mercado interno

Reconoció el TAS que en el mercado del fútbol existe un acuerdo entre los clubes, los cuales fungen como empresas, que están afiliadas a asociaciones federativas. Por ello, la Comisión Europea catalogó a la FIFA como una "asociación de asociaciones de empresas" (TAS, 2017, RFC Seraing vs. FIFA, num. 135, p. 25). Frente al segundo requisito mencionado, consideró el tribunal que los TPO pertenecen al mercado de los operadores financieros, diferente al de los clubes o los jugadores, quienes están en el mercado de una actividad deportiva, el fútbol, por lo cual no se afectaría el comercio regional. Y ante la tercera condición, el TAS creyó que no había suficientes pruebas allegadas por la parte recurrente, que permitieran verificar una restricción, prevención o distorsión en la competencia del mercado interior de la trasnferencia y cesión de derechos de los jugadores (TAS, 2017, RFC Seraing vs. FIFA, nums. 135-137, pp. 25-26).

Así mismo, el TAS desestimó la existencia de un abuso de posición dominante (TAS, 2017, RFC Seraing vs. FIFA, nums. 139-144, pp. 27-28; UE, 2007, art. 102), o que se vunerara la libertad profesional y laboral, la libertad de empresa, la libertad económica y el derecho de propiedad (TAS, 2017, RFC Seraing vs. FIFA, nums. 145-161, pp. 28-30)

Se colige que las enmiendas al RETJ aprobadas por la FIFA, tienen por objetivo evitar la afectación de la organización de las competiciones oficiales, debido a la probada presión que ejercían los TPO sobre los clubes para que sus jugadores fueran incluidos en las nóminas titulares, así como el amaño de partidos, el blanqueamiento de capitales, el soborno, entre otras conductas ilegales. Así, para el TAS, el poder disciplinario ejercido por la FIFA de prohibir las actividades de los TPO en las transferencias y cesiones de derechos de jugadores es legalmente válido, reafirmando que su competencia sancionadora se suscribe exclusivamente sobre los clubes y jugadores afiliados a la FIFA.

Queda para otros trabajos analizar si la implementación de estas modificaciones ha dado los resultados esperados respecto a los objetivos planteados por la FIFA, los cuales se enrarecen tras los escándalos de corrupción relacionados con los denominados Panama Papers (2015) y el FIFA Gate (2015-2016), los cuales vincularon empresas como Torneos y Competencias, Valente Corp, Somerton Ltd., Traffic Sports USA Inc., Traffic Sports International, Full Play Group SA, entre otras.

Un fenómeno que también cabe revisar es en qué manera los antiguos TPO se han convertido en inversionistas, accionistas mayoritarios y propietarios de clubes deportivos, vulnerando su autonomía institucional, al presionar o direccionar sus actividades competitivas y financieras, en beneficio de sus intereses económicos particulares. Esta fue un argumento presentado por el club belga en el citado proceso ante el 
TAS, ante el cual señaló que en caso de que los TPO trasmuten a esas figuras, quedarán sujetos a la reglamentación y la supervisión de la FIFA (TAS, 2017, RFC Seraing vs. FIFA, num. 106, pp. 19-20).

\section{¿El futbolista transferido internacionalmente debe ser considerado como un tercero?}

El RETJ en el 2014 no fue claro en establecer si el jugador es o no un tercero frente a la propiedad de sus derechos económicos, ya que no hacía referencia a este, sino a los dos clubes involucrados en el traspaso, así como a los clubes en lo que estuvo inscrito el futbolista. La primera interpretación que hicieron algunos dirigentes de la FIFA, juristas, directivos de clubes y agentes, es que el futbolista no podía ser considerado como un tercero, por lo que no debería recibir una retribución adicional a la transferencia de sus derechos deportivos o en un contrato de cesión de sus derechos económicos. Se argumentaba que, así como se estaba depurando las malas prácticas que había traído consigo los TPO en el mercado de pases y en la afectación de la autonomía de los clubes, también era necesario excluir al jugador sujeto de la transferencia, para evitar una mutación de los TPO a través del mismo jugador.

El debate fue amplio, ya que las voces que consideraban que el jugador no es un tercero frente a sus derechos económicos, se incrementaron. Se considera que, en primera medida, el jugador es el objeto de una transferencia de derechos, siendo la fuente primaria ya que sin su consentimiento expreso al momento que se celebre el negocio, los derechos del futbolista no podrían ser cedidos a ningún club:

(...) nunca el futbolista puede ser considerado un tercero en razón de que para perfeccionar la transferencia se requiere su consentimiento y además hay que realizar una interpretación finalista de la norma. En tal sentido, no caben dudas que lo que se buscó prohibir en el año 2014 es que un particular -un inversor- sea titular de derechos económicos. La prohibición de la FIFA va encaminada a evitar que los derechos económicos estén en cabeza de un particular, pero no del futbolista. Por ello, siempre consideramos que el jugador no era un tercero en los términos de los arts. 18 bis y 18 ter del RETJ de la FIFA (Gerbaundo, 2019).

Es incorrecto interpretar, que conforme a la normativa del RETJ, el jugador es un tercero por cuanto tiene capacidad plena o tutelada de contraer derechos y obligaciones para celebrar operaciones jurídicas, bien sea como cedente o cesionario de derechos de contenido patrimonial ante una eventual transferencia, además de ser la persona que genera los derechos económicos en cuestión:

(...) siendo el jugador el medio por el cual se origina el derecho, a tal grado que lo que se traspasa es su actividad -que solo él exclusivamente la ejerce-, no podemos comprender como quien origina un derecho, se pueda considerar después ajeno a él y sin poder ejercer por su cuenta la extensión del beneficio de la consecuencia económica de ese, su propio derecho inicial. Un club con el que no tiene contrato, es decir un tercero en relación al jugador, podría obtener con el consentimiento de éste un derecho económico sobre su actividad, pero a él, origen del derecho federativo que da lugar al derecho económico, no se le permite que amplíe su beneficio económico que sí lo puede obtener otro club con quien no tiene vínculo en la medida que ese club pase a tenerlo con su propio consentimiento (Nevascues, 2015).

Ante este panorama, la FIFA realizó el 1 de julio de 2019, a través de la circular 1679, una serie de enmiendas al RETJ, modificando, entre otros aspectos, la definición de tercero, señalando que es la "parte ajena al jugador siendo traspasado, a los dos clubes entre los cuales se traspasa al jugador, o a cualquiera de los clubes anteriores en los que el jugador estuvo inscrito previamente". Con ello se incluyó la expresión 
al jugador siendo traspasado (FIFA, 2019b, p. 1). De esta forma se aclaró la situación, al excluirse al jugador como tercero frente a la transferencia de sus derechos federativos y económicos, donde esta enmienda entró en vigencia a partir del $1^{\circ}$ de junio de 2019. Esta modificación fue explicada por la FIFA, al precisar que

\begin{abstract}
Se trata de un cambio en la definición que se ha introducido con el objeto de reflejar la jurisprudencia del Comité Disciplinario relacionada con una práctica reiterada por parte de los clubes, los cuales firman acuerdos con algunos de sus jugadores otorgándoles el derecho a recibir una compensación específica (una cantidad o un porcentaje) si en el futuro fueran transferidos a otro club. Estas cantidades prometidas a los jugadores se deben considerar parte de la remuneración acordada a los jugadores bajo las relaciones laborales con sus clubes y dichos acuerdos no deben considerarse violación alguna de la normativa de la FIFA en materia de propiedad de los derechos económicos de jugadores por parte de terceros.
\end{abstract}

La citada circular incluyó en el Anexo 2 una complementación al listado de definiciones de los términos utilizados en el RETJ, el cual contenía 16 definiciones y que ahora es de 23. Entre estos términos, se definen la transferencia internacional y la transferencia nacional, siendo la primera asumida como el "traslado de la inscripción de un jugador de una asociación a otra asociación" (FIFA, 2019b, pp. 4-5).

Se considera que, si bien es un concepto básico que concuerda con la naturaleza de este tipo de transacción comercial internacional, ofrece varios vacíos interpretativos conforme a lo que se ha detallado en este trabajo, ya que no se estructura con completitud el objeto contractual, las partes concurrentes y las relaciones jurídicas que surgen de este negocio jurídico plurilateral y sui generis.

\title{
V. Conclusiones
}

El fútbol profesional pertenece a la industria del entretenimiento, siendo un enorme y creciente mercado de connotaciones internacionales que involucra a los clubes y jugadores afiliados a las respectivas federaciones, los cuales obtienen ingresos por derechos de imagen, publicidad, merchandising, derechos de televisión, venta de boletería y, especialmente, por las transferencias y cesiones de los derechos de los jugadores.

Los derechos de los futbolistas involucran tanto los derechos federativos como los derechos económicos, son un activo esencial para la subsistencia y proyección empresarial de los clubes deportivos, en especial para aquellos que corresponden a países con menor grado de desarrollo, como los latinoamericanos. Las transferencias internacionales de estos estos derechos son reguladas por la FIFA a través de sus estatutos y reglamentos, teniendo como protagonistas a los futbolistas profesionales y a los clubes deportivos.

Los derechos federativos son entendidos como la potestad de un club de inscribir a un jugador en una competición oficial y organizada a través de la asociación a la que está afiliado. Se tratan de negocios jurídicos que parten de su indivisibilidad, ya que le pertenecen de forma exclusiva a los clubes.

Los derechos económicos son los beneficios económicos que se desprenden de la transferencia o cesión de los derechos federativos. La FIFA permite la cotitularidad de los derechos económicos tanto a los clubes como a los mismos jugadores sujeto de la transferencia. Hasta el 2015, permitía la cotitularidad a los terceros, fuesen personas naturales o jurídicas ajenas al fútbol. Sin embargo, con las enmiendas aprobadas ese año al Reglamento sobre el Estatuto y la Transferencia de los Jugadores (RETJ), la FIFA prohibió su intervención, y con ello se prohibió el accionar de los Third Party Ownership (TPO) en estas transacciones, figura 
que había tenido gran acogida en el entorno sudamericano, específicamente en Argentina y que luego fue desarrollada intensivamente en Brasil.

Los contratos internacionales celebrados por los clubes y los jugadores con la intervención terceros ajenos al control de la FIFA, son inválido a partir del 1 de mayo de 2016 (FIFA, 2019a). Por supuesto, esto representa un impacto profundo en el mercado del fútbol, tal como se ha configurado en las últimas cuatro décadas, con una especial incidencia en ámbito regulatorio de los clubes deportivos latinoamericanos, así como en la dinámica financiera de las inversiones en los clubes, y también en las trasferencias y las cesiones de los derechos económicos de los jugadores

Las prohibiciones emanadas de las enmiendas aprobadas por la FIFA al RETJ fueron revisadas por el Tribunal de Arbitramento Deportivo (TAS), que mediante laudo del 2019 consideró que la normativa legalmente válida frente al derecho de la Unión Europea, ya que no vulnera sus disposiciones en materia de libre circulación de capitales, libre circulación de trabajadores y de prestación de servicios, así como del derecho de la competencia. De igual forma, el TAS desestimó que se presentara un abuso de posición dominante, o se afectara la libertad profesional y laboral, la libertad de empresa, la libertad económica y el derecho de propiedad.

La fundamentación jurídica del tribunal arbitral tuvo en cuenta que los objetivos de la FIFA con estas modificaciones normativas consisten en evitar el trato discriminatorio en la transferencia de los futbolistas, la salvaguarda de la estabilidad contractual de los futbolistas, la independencia y autonomía de los clubes y los jugadores al momento de celebración de transferencias y cesiones de derechos económicos, así como preservar la justicia y trasparencia del entorno futbolístico.

A raíz de las enmiendas del 2015 al RETJ, surgió la controversia interpretativa si los jugadores que son sujeto de las transferencias de sus derechos, deben o no ser considerados como terceros frente a la naturaleza contractual del negocio. Por ello, la FIFA decidió en el 2019 aprobar unas modificaciones al Reglamento, precisando que el jugador en cuestión no debe ser considerado como un tercero. Se trata de una postura normativa que se comparte ya que es el jugador una de las diversas partes que integran un contrato de transferencia o cesión de sus derechos federativos y económicos, dependiendo de su consentimiento para la validez de dicho negocio jurídico.

Por último, es pertinente y urgente fortalecer los controles y mecanismos de inspección, control y vigilancia por parte de las autoridades de orden nacional en materia deportiva, así como por parte de las asociaciones y la FIFA, sobre los contratos celebrados acerca de transferencias y cesiones de derechos de futbolistas, procurando disminuir la opacidad de la naturaleza de los inversionistas, accionistas y propietarios de los clubes, así como como determinar el origen de los recursos utilizados en estos negocios jurídicos, su trasparencia corporativa y fiscal, así como el grado de influencia que todavía ejercen los terceros sobre los jugadores, los clubes deportivos y las asociaciones. 


\section{Referencias Bilbiograficas}

Asunto C-19/02, Dieter Kraus vs. Land Baden-Wuerttemberg, 1993 TJCE I-1663

Asunto C-415/93, Union Royale Belge des Sociétés de Football Association ASBL vs. Jean-Marc Bosman Royal Club Liégeois SA vs. Jean-Marc Bosman, SA d'économie mixte sportive de L'Union Sportive du Littoral de Dunkerque, Union Royale Belge des Sociétés de Football Association ASBL y Union des Associations Européennes de Football (UEFA) - Union des Associations Européennes de Football (UEFA) vs. Jean-Marc Bosman, 1995 TJCE I-5082

Auletta, M. (2014). Derecho Deportivo / Comentarios y Reflexiones. Philos Iuris.

Auletta, M. (2014). Derechos y beneficios económicos en el fútbol profesional. Breve análisis jurídico de las transferencias de futbolistas profesionales y de algunos negocios vinculados a las mismas. Revista Republicana, 1(16), 17-43.

Batista, A. (2017). Razones por las que el TAS confirmó la prohibición de los TPO. https://www.gherasociados. com/es/razones-por-las-que-el-tas-confirmo-la-prohibicion-de-los-tpo/

Bruera, P. (2018). Derechos económicos (TPO: Third Part Ownership). https://www.derechosdelfutbol.com/ derechos-economicos

Cazorla, L. (2014). La UEFA y la pretendida nulidad de la "cláusula Courtois". https://iusport.com/art/1998/ la-uefa-y-la-pretendida-nulidad-de-la-clausula-courtois

Cazorla, L. (2016). Fondos de Inversión y fútbol profesional (I): Derechos federativos y Derechos económicos sobre un futbolista. http://luiscazorla.com/2013/10/fondos-de-inversion-y-futbol-profesional-i-derechos-federativos-y-derechos-economicos-sobre-un-futbolista/

Corte Constitucional [CC], 17 de julio, 1998, MP: A. Martínez Caballero, Sentencia T-371/98, [Col.].

Corte Constitucional [CC], 18 de junio, 1998, MP: A. Martínez Caballero, Sentencia T-302/98, [Col.].

Corte Constitucional [CC], 3 de julio, 1997, MP: A. Martínez Caballero, Sentencia C-320/97, [Col.].

Corte Constitucional [CC], 4 de noviembre, 1994, MP: E. Cifuentes Muñoz, Sentencia T-408/94, [Col.].

Crespo, J., \& Frega, R. (2015). Nuevos comentarios al reglamento FIFA : con análisis de jurisprudencia de la DRC y del TAS. Dykinson.

Decreto 410 de 1971. Por el cual se expide el Código de Comercio. 27 de marzo, 1971. DO núm. 33.339 (Col.).

Di Angelo, C. (2015). Validez de la cesión de derechos económicos. Derecho federativos. http://cristiandiangelo.blogspot.com/2015/12/validez-de-la-cesion-de-derechos.html

Federación Internacional de Fútbol Asociado [FIFA]. (2014). Circular n. 1464. https://img.fifa.com/image/ upload/rui2hdrmosqjcatbmybf.pdf

Federación Internacional de Fútbol Asociado [FIFA]. (2016, 26 de febrero). Estatutos de la FIFA. https://img. fifa.com/image/upload/azwxwekfmx0nfdixwv1m.pdf

Federación Internacional de Fútbol Asociado [FIFA]. (2019a). Reglamento sobre el Estatuto y la Transferencia de Jugadores. https://resources.fifa.com/image/upload/reglamento-sobre-el-estatuto-y-la-transferencia-de-jugadores-junio-2019.pdf?cloudid=05g4zec5mrcndsl8h3ip

Federación Internacional de Fútbol Asociado [FIFA]. (2019b). Enmiendas al reglamento sobre el Estatuto y la Transferencia de Jugadores. https://resources.fifa.com/image/upload/no-1679-amendments-juneand-october-2019.pdf?cloudid=hzmhs59uxezr1gpgsylq

Federación Internacional de Fútbol Asociado [FIFA]. (2020a). Global Transfer Market Report 2019. Men Professional Football. A Review of International Football Transfers Worldwide. https://img.fifa.com/image/ upload/x2wrqjstwjoailnncnod.pdf 
Federación Internacional de Fútbol Asociado [FIFA]. (2020b). Global Transfer Market Report 2019. Women Professional Football. A Review of International Football Transfers Worldwide. https://resources.fifa.com/ image/upload/global-transfer-market-report-2019-women.pdf?cloudid=dm3mxtl95tjrufvuohwn

Gerbaundo, G. E. (2019). Los derechos económicos. El futbolista como titular de sus propios derechos económicos. https://dpicuantico.com/area_diario/doctrina-en-dos-paginas-diario-dpi-suplemento-derecho-del-deporte-nro-18-12-08-2019/

Ley 181 de 1995. Por el cual se dictan disposiciones para el fomento del deporte, la recreación, el aprovechamiento del tiempo libre y la Educación Física y se crea el Sistema Nacional del Deporte. 18 de enero, 1995. DO núm. 41.679 (Col.)

Ley 84 de 1873. Código Civil de los Estados Unidos de Colombia. 26 de mayo, 1873. DO núm. 2.867 (Col.)

Nadal, M. (2014, 17 de enero). Derechos económicos y fondos de inversión en fútbol: ideas básicas. https:// iusport.com/art/1450/derechos-economicos-y-fondos-de-inversion-en-futbol-ideas-basicas

Nevascues, H. (2015, 28 de junio). Artículo 18 Ter y los TPO: ¿es el jugador un tercero? https://iusport.com/ art/8274/articulo-18-ter-y-los-tpo-es-el-jugador-un-tercero-

Palazzo, I. (2014, 26 de diciembre). Los legítimos titulares de derechos económicos de los futbolistas tras la abolición de los fondos de inversión. https://iusport.com/art/4218/los-legitimos-titulares-de-derechos-economicos-de-los-futbolistas-tras-la-abolicion-de-los-fondos-de-inversion

RFC Seraing vs. Federación Internacional de Fútbol Asociado (FIFA), TAS 2016/A/4490 (2017)

Superintendencia de Sociedades. (2020). Comportamiento financiero de los clubes de fútbol en 2019. https:// www.supersociedades.gov.co/Noticias/Publicaciones/Revistas/2020/Informe-de-Futbol-2019.pdf

Unión Europea [UE], Carta de los derechos fundamentales de la Unión Europea, 2012/C 326/02, 7 de diciembre, 2000.

Unión Europea [UE], Versiones consolidadas del Tratado de la Unión Europea y del Funcionamiento de la Unión Europea, 2012/C 326/01, 13 de diciembre, 2007. 